\title{
METODOLOGIA FUZZY APLICADA À AVALIAÇÃO DO AUMENTO DA TEMPERATURA CORPORAL EM FRANGOS DE CORTE
}

\author{
Leonardo Schiassi ${ }^{1}$, Tadayuki Yanagi Junior ${ }^{2}$, Leandro Ferreira ${ }^{3}$, Flávio Alves \\ Damasceno $^{3}$, Silvia de Nazaré Monteiro Yanagi ${ }^{4}$
}

\begin{abstract}
RESUMO
Um sistema de inferência fuzzy foi desenvolvido, com o objetivo de predizer o aumento da temperatura corporal (ATC) em frangos de corte $(2,8 \pm 0,1 \mathrm{~kg})$ aos 90 minutos de exposição térmica, sob diferentes condições de estresse térmico agudo. O sistema fuzzy foi estruturado com base em três variáveis de entrada: temperatura do ambiente $\left(t_{b s}\right)$, umidade relativa (UR) e velocidade do ar $(V)$, sendo que o nível de desconforto térmico, sofrido pelas aves, foi classificado por meio da variável de saída, ATC. A inferência fuzzy foi realizada por meio do método de Mamdani, que consistiu na elaboração de 45 regras e a defuzificação por meio do método do Centro de Gravidade. As operações fuzzy foram realizadas no ambiente computacional MATLAB ${ }^{\circledR}$ 6.5. Além de fornecer resultados promissores, a metodologia fuzzy apresenta potencial considerável de aplicação na área de ambiência avícola.
\end{abstract}

Palavras-chave: modelagem, inferência fuzzy, estresse térmico agudo.

\section{ABSTRACT}

\section{Fuzzy methodology applied to the evaluate body temperature increase of broiler chickens}

A fuzzy inference system was developed to predict body temperature rise (BTR) of broiler chickens $(2.8 \pm 0.1 \mathrm{Kg})$ after 90 -min thermal exposure under different acute thermal stress condition. The structured fuzzy system was based on three input variables: room temperature $\left(t_{d b}\right)$, relative humidity (rh) and air velocity (V), where the level of thermal discomfort felt by the chickens was classified through the output variable, BTR. Fuzzy inference was performed through the Mamdani's method that consisted of elaborating 45 rules and the defuzzification by Center of Gravity method. The fuzzy operations were performed using MATLAB ${ }^{\circledR}$ 6.5. Besides providing promising results, the fuzzy methodology shows considerable potential to be applied in poultry environment area.

Keywords: modeling, fuzzy inference, acute thermal stress.

Recebido para publicação em 27.08.2007

${ }^{1}$ Graduando em Engenharia Agrícola, Departamento de Engenharia, UFLA, leoschiassi@ yahoo.com.br.

${ }^{2}$ Eng. Agrícola, Dr., Professor do Departamento de Engenharia, UFLA, yanagi@ufla.br

${ }^{3}$ Mestrando em Engenharia Agrícola - Construções e Ambiência, UFLA, flavioufla@ yahoo.com.br.

${ }^{4}$ Pós-Doutoranda, Dra., Departamento de Engenharia, UFLA, smonteiro@ufla.br. 


\section{INTRODUÇÃO}

O potencial produtivo dos animais está diretamente relacionado ao tipo de manejo utilizado, que pode ser quantificado pelas condições do ambiente térmico, como radiação solar, temperatura do ar ( $\left.\mathrm{t}_{\mathrm{bs}}\right)$, umidade relativa (UR) e velocidade do ar (V), possibilitando assim, quantificar o nível de conforto ou desconforto térmico.

Neste contexto, as instalações zootécnicas contribuem de forma primordial para a obtenção de um ambiente térmico adequado à produção animal, cujos limites são caracterizados pela zona de termoneutralidade (CURTIS, 1983). Entretanto, quando as condições ambientais não estão dentro do limite adequado, que é caracterizado pela zona de termoneutralidade, 0 ambiente torna-se desconfortável, porém, o organismo animal tenta ajustar-se, fisiologicamente, para manter sua homeotermia, absorvendo ou dissipando calor, o que pode resultar em perdas produtivas em diferentes níveis.

O estresse térmico pode ser crônico ou agudo. No caso do estresse crônico, os animais ficam submetidos a condições térmicas desconfortáveis, durante um longo intervalo de tempo, propiciando a aclimatação, enquanto que, no estresse agudo, 0 ambiente térmico varia bruscamente, atingindo níveis de desconforto consideráveis e impossibilitando a aclimatação adequada dos animais (CURTIS, 1983). Este caso está diretamente relacionado às ondas de calor, que atingem diversas áreas no globo, inclusive o Brasil, causando danos significativos ao desempenho animal, podendo inclusive ocorrer perdas no próprio plantel, causando altos prejuízos ao setor avícola (YANAGI JUNIOR, 2002).

Para frangos de corte, o conforto térmico é caracterizado por intervalos de $t_{b s}$ entre $15^{\circ} \mathrm{C}$ e $25^{\circ} \mathrm{C}$ (CURTIS, 1983), UR entre $50 \%$ e $60 \%$ (DONALD, 1998), e $\mathrm{V}$ em torno de 0,2 m.s ${ }^{-1}$ aproximadamente, no inverno e 0,5 m. s $^{-1}$ no verão (CURTIS, 1983).

Apesar dos valores de $t_{b s}$, UR e V serem importantes na avaliação do ambiente térmico, o uso de índices ambientais são mais adequados como, por exemplo, o índice de temperatura e umidade (ITU) desenvolvido por Thom (1959) e o índice de temperatura, umidade e velocidade do ar (ITUV) proposto por Tao \& Xin (2003), entre outros.

No caso específico do ITUV (equação 1), que avalia $o$ efeito do estresse térmico agudo e a taxa de mortalidade em frangos de corte, o nível de estresse foi quantificado e classificado com base no aumento da temperatura corporal, aos 90 minutos de exposição térmica e no fim de cada teste $\left(\Delta \mathrm{t}_{90}\right.$ e $\Delta \mathrm{t}_{\text {final }}$, respectivamente).

$$
I T U V=\left(0,8 \bar{f}_{b s}+0,1 \bar{f}_{b u}\right) \cdot V^{-0.058}(0,2 \leq \mathrm{V} \leq 1,2)
$$

em que,

$\mathrm{t}_{\mathrm{bs}}=$ temperatura de bulbo seco do ar $\left({ }^{\circ} \mathrm{C}\right)$;

$\mathrm{t}_{\mathrm{bu}}=$ temperatura de bulbo úmido do $\operatorname{ar}\left({ }^{\circ} \mathrm{C}\right)$ e

$\mathrm{V}=$ velocidade do $\operatorname{ar}\left(\mathrm{m} . \mathrm{s}^{-1}\right)$

Tendo como ponto de partida as variáveis ambientais, processos de decisão devem ser tomados para que o ambiente seja adequado à produção. Neste contexto, a aplicação da lógica fuzzy é uma alternativa interessante na tomada de decisão, possibilitando, assim, o controle do ambiente térmico no interior de galpões para produção de frangos de corte. A utilização da lógica fuzzy permite uma generalização de informações, que estão associadas ao gerenciamento de incertezas. A lógica fuzzy, também chamada de conjuntos nebulosos ou difusos, é uma extensão da lógica clássica e foi estudada primeiramente por Lofti Zadeh, na Universidade de Califórnia, em 1965 (ZADEH, 1965).

Um conjunto clássico é representado por sua função característica, em que $\mu_{A}(x)$ representa 0 grau de pertinência com que 0 elemento $x$ assume no conjunto $A$, conforme especificado na equação

$$
\mu_{A}(x)=\left\{\begin{array}{l}
1, \text { se } x \in A \\
0, \text { se } x \notin A
\end{array}\right.
$$


Observa-se que, nos conjuntos clássicos, - elemento pode assumir apenas dois valores de pertinência (aspecto bivalente), em que o meio é excluído. Nos conjuntos fuzzy, ocorre a flexibilização do intervalo de pertinência, na qual a transição entre pertinência é gradual, em vez de brusca. A função característica dos conjuntos fuzzy é dada pela equação

$\mu_{\mathrm{A}}(\mathrm{x})=[0,1]$

A metodologia fuzzy está baseada na construção dos conjuntos fuzzy, que representam as informações em função de termos lingüísticos. Deste modo, os conjuntos fuzzy expressam conceitos vagos, assim como a linguagem natural (Klir \& Yuan, 1995). Um conjunto fuzzy é representado por meio de uma função de pertinência, que indica $o$ valor de pertinência, que um determinado elemento assume com relação a um conjunto. As formas para as funções de pertinência são arbitrárias, sendo que as mais utilizadas são as triangulares, trapezoidais e gaussianas (ORTEGA, 2001). As operações dos conjuntos fuzzy são obtidas a partir da teoria dos conjuntos clássicos, em que se tem o estudo das operações fundamentais (união, intersecção e complemento), números fuzzy e o princípio da extensão (KLIR \& YUAN, 1995; TANAKA, 1997; PEDRYCZ \& GOMIDE, 1998).
Um sistema fuzzy é constituído por variáveis de entrada e saída. Para cada variável, são atribuídos conjuntos fuzzy que as caracterizam, sendo que, para cada conjunto fuzzy, é criada uma função de pertinência. Em seguida, é realizada a inferência fuzzy, que consiste na construção do sistema de regras, caracterizando as variáveis de entrada e saída com seus respectivos conjuntos fuzzy. O sistema de regras pode ser construído com base em bancos de dados e opiniões de especialistas. Cada regra é composta por conectivos lógicos (se, e, ou, então), como, por exemplo,

\section{Regra: SE x é A E y é B ENTÃO z é C}

em que,

A, B e C são conjuntos fuzzy.

Segundo Mendel (1995), para o desenvolvimento computacional de um sistema fuzzy pode-se utilizar um software capaz de realizar todos os procedimentos necessários à sua construção, sendo que a avaliação computacional é caracterizada pelas etapas de fuzificação (transforma as variáveis de entrada em funções de pertinência), inferência (realiza o raciocínio fuzzy com base num sistema de regras, promovendo a saída fuzzy) e defuzificação (realiza a tradução do valor lingüístico em valor numérico), conforme ilustrado na Figura 1.

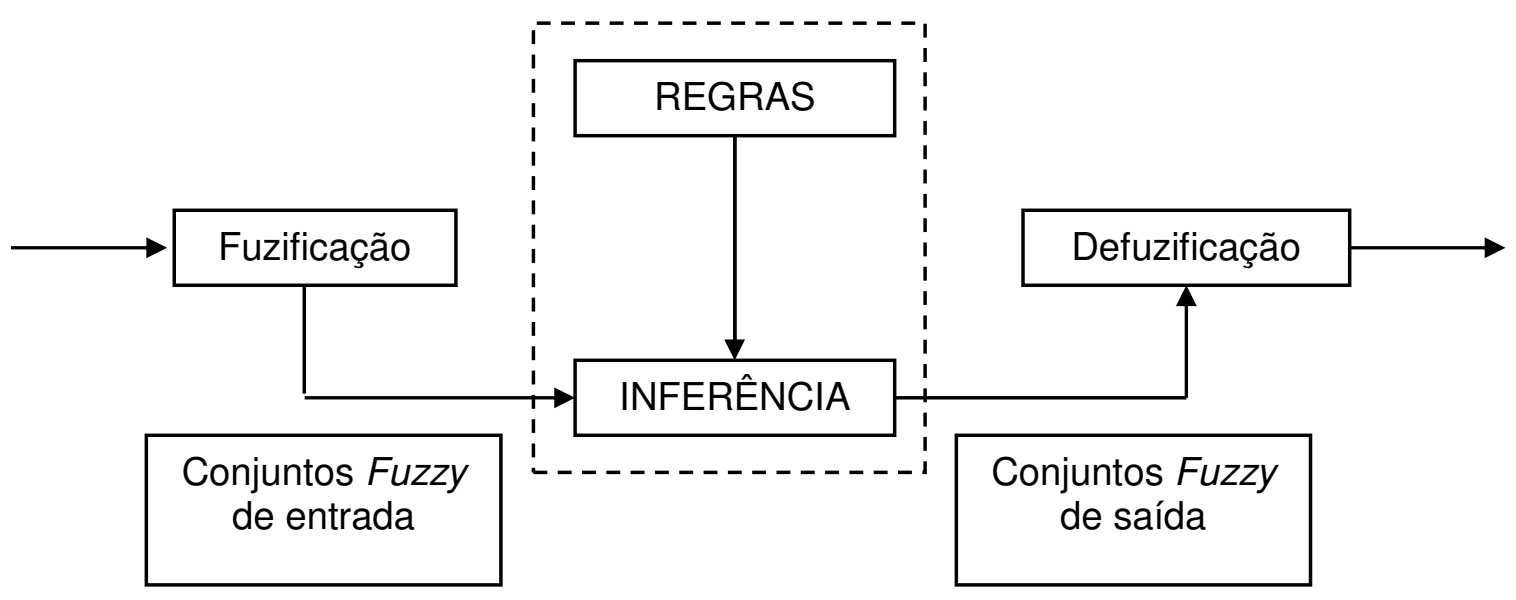

Figura 1. Desenvolvimento computacional fuzzy 
A metodologia fuzzy tem sido utilizada em várias áreas, como análises de dados, sistemas especialistas, controle e otimização, controle de aeronaves e biomedicina (Ribacionka, 1999; Lopes, 1999; Ortega, 2001; Weber \& Klein, 2003). $\mathrm{Na}$ área de ambiência animal, várias aplicações indicam o seu potencial de uso, como o estudo do conforto térmico em aves (CHAO et. al., 2000; GATES et. al., 2001; AMENDOLA et al., 2004; AMENDOLA et al., 2005; OLIVEIRA et al., 2005, YANAGI JUNIOR et al., 2006) e suínos (QUEIROZ et al., 2005), além de ser usado para detecção de cio em vacas leiteiras (FIRK et al., 2003, FERREIRA et al., 2006).

Portanto, no presente trabalho, objetivou-se desenvolver um sistema de auxílio à tomada de decisão, com base na metodologia dos conjuntos fuzzy, para predizer o aumento máximo da temperatura corporal (ATC) aos 90 minutos de exposição térmica, sob diferentes condições de estresse térmico agudo em frangos de corte.

\section{MATERIAL E MÉTODOS}

O desenvolvimento do sistema fuzzy baseou-se na pesquisa desenvolvida por
Tao \& Xin (2003), que propuseram o ITUV e avaliaram o ATC, sob diferentes condições de estresse térmico agudo em frangos de corte $(2,8 \pm 0,1 \mathrm{Kg})$ da linhagem Ross x Ross.

Para o desenvolvimento do sistema fuzzy foram definidas, como variáveis de entrada: temperatura de bulbo seco do ar $\left(t_{b s}\right)$, umidade relativa (UR) e velocidade do ar (V). Com base nas variáveis de entrada, o sistema fuzzy (Figura 2) prediz o ATC, avaliando o nível de desconforto térmico por meio da classificação do ambiente. A análise foi desenvolvida com o auxílio do software MATLAB ${ }^{\circledR}$ 6.5, sendo utilizado 0 método de inferência fuzzy de Mamdani (Amendola \& Souza, 2004) que, como resposta, traz um conjunto fuzzy originado da combinação dos valores de entrada com os seus respectivos graus de pertinência através do operador mínimo e em seguida pela superposição das regras por meio do operador máximo. A defuzificação foi feita, utilizando-se o método do Centro de Gravidade (Centróide ou Centro de Área), que considera todas as possibilidades de saída, transformando o conjunto fuzzy, originado pela inferência, em valor numérico, conforme proposto por Amendola \& Souza ( 2004).

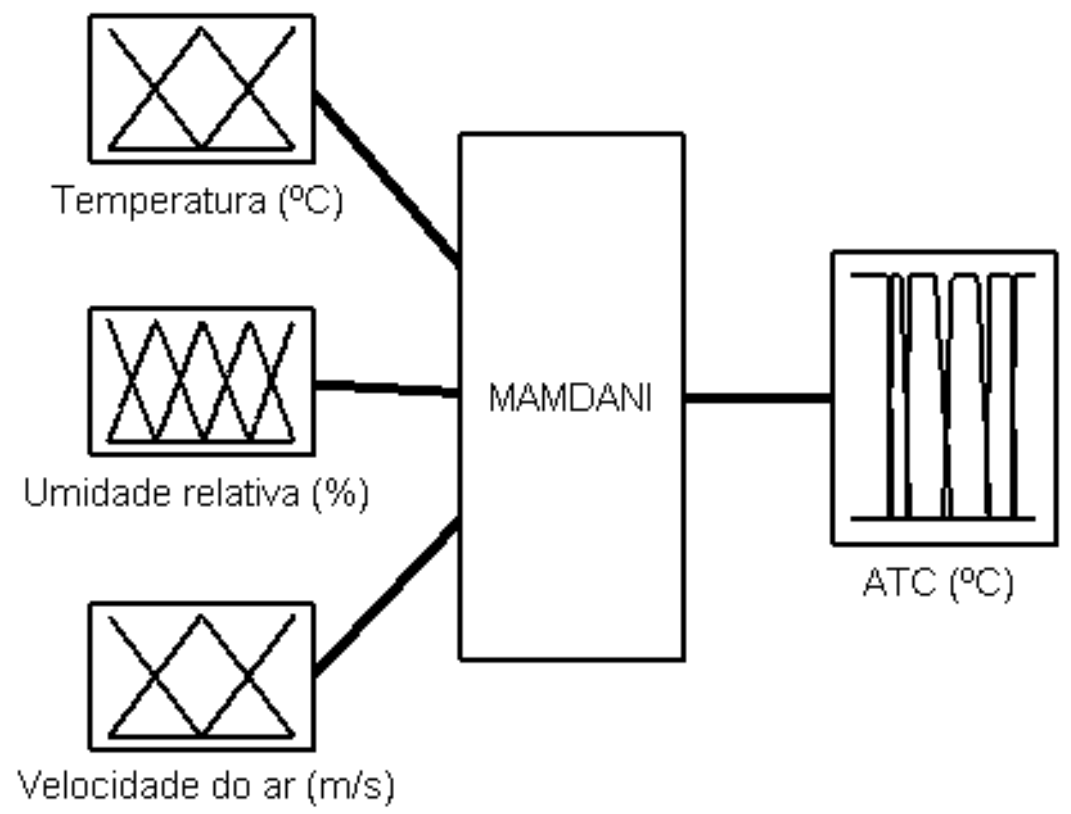

Figura 2. Esquema ilustrativo do sistema fuzzy 
Considerando que as principais variáveis, que influenciam o ambiente térmico no interior de galpões para produção de frangos de corte, são a $t_{b s}$, UR e V, bem como sua disponibilidade na literatura, optou-se por utilizar estas variáveis como entradas para o sistema.

Os intervalos de $\mathrm{t}_{\mathrm{bs}}, \mathrm{UR}$ e $\mathrm{V}$, adotados no desenvolvimento do sistema fuzzy proposto, foram escolhidos com base no experimento desenvolvido por Tao \& Xin (2003), que avaliou o efeito do estresse agudo sobre o desempenho de frangos de corte.
Os conjuntos fuzzy das variáveis de entrada são mostrados no Quadro 1.

Os intervalos admitidos para as variáveis de entrada $t_{b s}$, UR e V estão, graficamente, representados pelas curvas de pertinência triangulares, pois representam melhor 0 comportamento dos dados de entrada e por serem as mais usadas, de acordo com a literatura (AMENDOLA et al., 2004; FERREIRA et al., 2006; YANAGI JUNIOR et al., 2006), conforme ilustrado na Figura 3.

Quadro 1. Conjuntos Fuzzy para as variáveis de entrada

\begin{tabular}{|c|c|c|}
\hline Tipo de variável & Variáveis & Conjuntos fuzzy \\
\hline \multirow{11}{*}{ Entrada } & \multirow{3}{*}{ Temperatura do ar $\left({ }^{\circ} \mathrm{C}\right)$} & T1 $[35 ; 38]$ \\
\hline & & T2 [35; 41] \\
\hline & & T3 [38; 41] \\
\hline & \multirow{5}{*}{ Umidade relativa (\%) } & UR1 [29; 37] \\
\hline & & UR2 [29; 45] \\
\hline & & UR3 [37; 52] \\
\hline & & UR4 $[45 ; 60]$ \\
\hline & & UR5 $[52 ; 60]$ \\
\hline & \multirow{3}{*}{ Velocidade do ar $\left(m \cdot s^{-1}\right)$} & V1 $[0,2 ; 0,7]$ \\
\hline & & V2 $[0,2 ; 1,2]$ \\
\hline & & V3 $[0,7 ; 1,2]$ \\
\hline
\end{tabular}

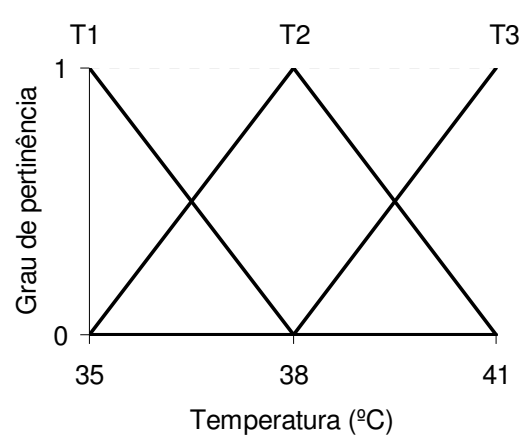

(a)

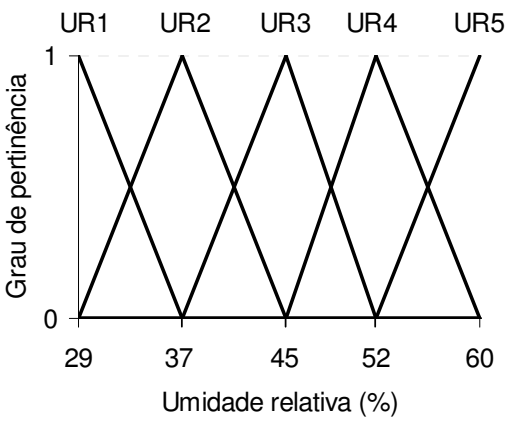

(b)

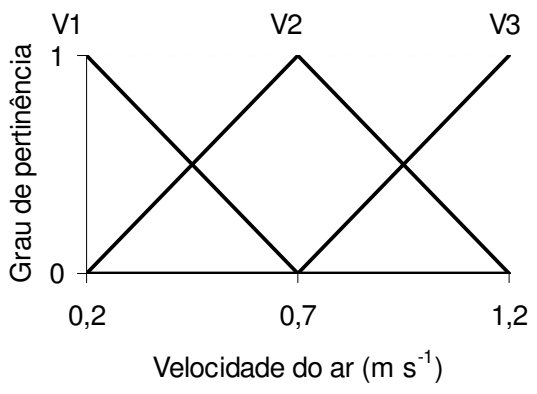

(c)

Figura 3. Curvas de pertinência para as variáveis de entrada (a), temperatura de bulbo seco do $\operatorname{ar}\left(\mathrm{t}_{\mathrm{bs}}\right)$, (b) umidade relativa (UR) e (c) velocidade do ar (V). 
As curvas de pertinência para a variável de saída (Figura 4) foram desenvolvidas com base na pesquisa conduzida por Tao \& Xin (2003), que propuseram a combinação fatorial de três níveis $\mathrm{t}_{\mathrm{bs}}\left(35,38\right.$ e $\left.41^{\circ} \mathrm{C}\right)$, dois níveis de temperaturas de ponto de orvalho $\left(t_{\mathrm{po}}\right)\left(19,4\right.$ e $\left.26,1^{\circ} \mathrm{C}\right)$ e três níveis de $\mathrm{V}\left(0,2,0,7\right.$ e $\left.1,2 \mathrm{~m} \mathrm{~s}^{-1}\right)$, totalizando dezoito condições térmicas distintas. A fim de melhorar a resposta do modelo, entretanto, cinco níveis de UR foram usados ao invés dos dois níveis, adotados pelos autores, para reduzir os erros associados às simulações.

A variável de saída, ATC, usada na construção do sistema fuzzy, permite a indicação direta do nível de estresse térmico agudo, sofrido pelos frangos de corte. Assim, os seguintes conjuntos fuzzy foram especificados, conforme mostrado no Quadro 2.

Os intervalos adotados para a variável de saída, ATC, foram caracterizados por curvas de pertinência do tipo trapezoidal (Figura 4), por representarem melhor o conjunto de dados e por resultarem nos menores valores de desvio padrão.

Considerando, como exemplo, o valor de $A T C=0,93^{\circ} \mathrm{C}$, que pertence a dois conjuntos fuzzy, ATC1 e ATC2, com grau de pertinência de 0,7 e 0,3 , respectivamente. $O$ processo de decisão admite que este valor de ATC pertença ao conjunto fuzzy ATC1, por apresentar maior grau de pertinência.

O sistema de regras (Quadro 3) foi desenvolvido com base nas combinações propostas por Tao \& Xin (2003), porém, aumentando o número de classificações para UR de dois para cinco, o que resultou na definição de 45 regras, sendo que, para cada regra, foi atribuído um fator de ponderação igual a 1 .

Quadro 2. Intervalo dos conjuntos fuzzy para as variável de saída aumento da temperatura corporal $($ ATC, $\stackrel{\circ}{ } \mathrm{C})$

\begin{tabular}{cc}
\hline Conjuntos fuzzy & Intervalo \\
\hline ATC1 & {$[0,0 ; 1,0]$} \\
ATC2 & {$[0,9 ; 1,4]$} \\
ATC3 & {$[1,3 ; 2,4]$} \\
ATC4 & {$[2,2 ; 3,3]$} \\
ATC5 & {$[3,2 ; 3,9]$} \\
ATC6 & {$[3,8 ; 4,4]$} \\
\hline
\end{tabular}

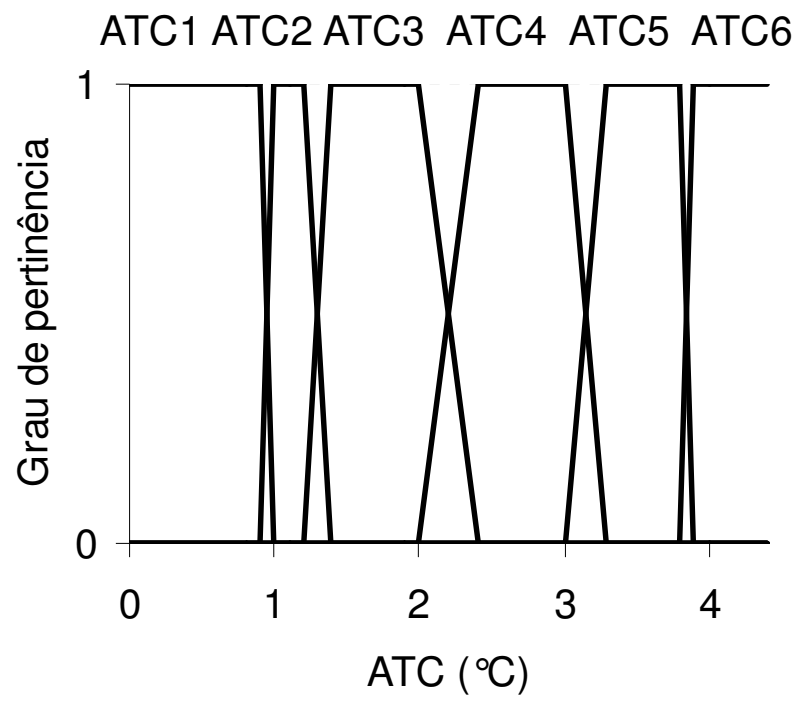

Figura 4. Curvas de pertinência para a variável de saída aumento da temperatura corporal (ATC). 
Quadro 3. Composição do sistema de regras utilizadas na inferência fuzzy para as características temperatura de bulbo seco do ar (T), umidade relativa (UR) e velocidade do ar (V)

\begin{tabular}{|c|c|}
\hline & Regra \\
\hline 1 & Se ( $t_{b s}$ é T1) E (UR é UR1) e (V é V1) Então (ATC é ATC3) \\
\hline 2 & Se ( $t_{b s}$ é T1) E (UR é UR1) e (V é V2) Então (ATC é ATC1) \\
\hline 3 & Se ( $t_{\text {bs }}$ é T1) E (UR é UR1) e (V é V3) Então (ATC é ATC1) \\
\hline 4 & Se ( $t_{b s}$ é T1) E (UR é UR2) e (V é V1) Então (ATC é ATC3) \\
\hline 5 & Se ( $t_{b s}$ é T1) E (UR é UR2) e (V é V2) Então (ATC é ATC1) \\
\hline 6 & Se ( $t_{\text {bs }}$ é T1) E (UR é UR2) e (V é V3) Então (ATC é ATC1) \\
\hline 7 & Se ( $t_{b s}$ é T1) E (UR é UR3) e (V é V1) Então (ATC é ATC3) \\
\hline 8 & Se ( $t_{b s}$ é T1) E (UR é UR3) e (V é V2) Então (ATC é ATC2) \\
\hline 9 & Se ( $t_{b s}$ é T1) E (UR é UR3) e (V é V3) Então (ATC é ATC1) \\
\hline 10 & Se ( $t_{\text {bs }}$ é T1) E (UR é UR4) e (V é V1) Então (ATC é ATC4) \\
\hline 11 & Se ( $\mathrm{t}_{\mathrm{bs}}$ é T1) E (UR é UR4) e (V é V2) Então (ATC é ATC2) \\
\hline 12 & Se ( $\mathrm{t}_{\mathrm{bs}}$ é T1) E (UR é UR4) e (V é V3) Então (ATC é ATC1) \\
\hline 13 & Se ( $t_{b s}$ é T1) E (UR é UR5) e (V é V1) Então (ATC é ATC3) \\
\hline 14 & Se ( $t_{b s}$ é T1) E (UR é UR5) e (V é V2) Então (ATC é ATC2) \\
\hline 15 & Se ( $t_{b s}$ é T1) E (UR é UR5) e (V é V3) Então (ATC é ATC2) \\
\hline 16 & Se ( $t_{b s}$ é T2) E (UR é UR1) e (V é V1) Então (ATC é ATC4) \\
\hline 17 & Se ( $t_{b s}$ é T2) E (UR é UR1) e (V é V2) Então (ATC é ATC3) \\
\hline 18 & Se ( $t_{b s}$ é T2) E (UR é UR1) e (V é V3) Então (ATC é ATC3) \\
\hline 19 & Se ( $t_{b s}$ é T2) E (UR é UR2) e (V é V1) Então (ATC é ATC3) \\
\hline 20 & Se ( $t_{b s}$ é T2) E (UR é UR2) e (V é V2) Então (ATC é ATC3) \\
\hline 21 & Se ( $t_{b s}$ é T2) E (UR é UR2) e (V é V3) Então (ATC é ATC3) \\
\hline 22 & Se ( $t_{b s}$ é T2) E (UR é UR3) e (V é V1) Então (ATC é ATC5) \\
\hline 23 & Se ( $t_{b s}$ é T2) E (UR é UR3) e (V é V2) Então (ATC é ATC4) \\
\hline 24 & Se ( $t_{b s}$ é T2) E (UR é UR3) e (V é V3) Então (ATC é ATC3) \\
\hline 25 & Se ( $t_{b s}$ é T2) E (UR é UR4) e (V é V1) Então (ATC é ATC4) \\
\hline 26 & 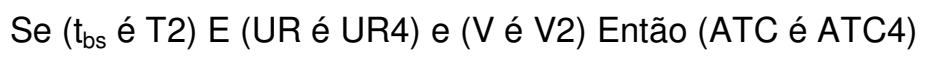 \\
\hline 27 & Se ( $t_{b s}$ é T2) E (UR é UR4) e (V é V3) Então (ATC é ATC3) \\
\hline 28 & Se ( $t_{b s}$ é T2) E (UR é UR5) e (V é V1) Então (ATC é ATC5) \\
\hline 29 & Se ( $t_{b s}$ é T2) E (UR é UR5) e (V é V2) Então (ATC é ATC4) \\
\hline 30 & Se ( $t_{b s}$ é T2) E (UR é UR5) e (V é V3) Então (ATC é ATC3) \\
\hline 31 & Se ( $t_{b s}$ é T3) E (UR é UR1) e (V é V1) Então (ATC é ATC5) \\
\hline 32 & Se ( $t_{b s}$ é T3) E (UR é UR1) e (V é V2) Então (ATC é ATC5) \\
\hline 33 & Se ( $t_{b s}$ é T3) E (UR é UR1) e (V é V3) Então (ATC é ATC5) \\
\hline 34 & Se ( $t_{b s}$ é T3) E (UR é UR2) e (V é V1) Então (ATC é ATC6) \\
\hline 35 & Se ( $\mathrm{t}_{\mathrm{bs}}$ é T3) E (UR é UR2) e (V é V2) Então (ATC é ATC6) \\
\hline 36 & Se ( $t_{b s}$ é T3) E (UR é UR2) e (V é V3) Então (ATC é ATC5) \\
\hline 37 & Se ( $t_{b s}$ é T3) E (UR é UR3) e (V é V1) Então (ATC é ATC6) \\
\hline 38 & Se ( $\mathrm{t}_{\mathrm{bs}}$ é T3) E (UR é UR3) e (V é V2) Então (ATC é ATC6) \\
\hline 39 & Se ( $t_{b s}$ é T3) E (UR é UR3) e (V é V3) Então (ATC é ATC6) \\
\hline 40 & Se ( $t_{b s}$ é T3) E (UR é UR4) e (V é V1) Então (ATC é ATC6) \\
\hline 41 & Se ( $t_{b s}$ é T3) E (UR é UR4) e (V é V2) Então (ATC é ATC5) \\
\hline 42 & Se ( $t_{b s}$ é T3) E (UR é UR4) e (V é V3) Então (ATC é ATC4) \\
\hline 43 & Se ( $t_{b s}$ é T3) E (UR é UR5) e (V é V1) Então (ATC é ATC6) \\
\hline 44 & Se ( $t_{b s}$ é T3) E (UR é UR5) e (V é V2) Então (ATC é ATC5) \\
\hline 45 & Se ( $t_{b s}$ é T3) E (UR é UR5) e (V é V3) Então (ATC é ATC4) \\
\hline
\end{tabular}


Quadro 4. Classificação do estado de conforto de frangos de corte submetidos a estresse térmico agudo, com base no aumento da temperatura corporal (ATC) das aves aos 90 minutos de exposição térmica

\begin{tabular}{cc}
\hline Classificação & Intervalo de ATC \\
\hline Leve desconforto (LD) & ATC $<1,0^{\circ} \mathrm{C}$ \\
Moderado desconforto (MD) & $1,0^{\circ} \mathrm{C}<$ ATC $\leq 2,5^{\circ} \mathrm{C}$ \\
Severo desconforto (SD) & $2,5^{\circ} \mathrm{C}<$ ATC $\leq 4,0^{\circ} \mathrm{C}$ \\
Extremo perigo de vida (EP) & ATC $>4,0^{\circ} \mathrm{C}$ \\
\hline
\end{tabular}

Os valores da ATC, calculados com base na equação proposta por Tao \& Xin (2003) (equação 4) e simulados pelo sistema fuzzy, foram usados para classificação do estado de conforto dos frangos de corte. Os valores do índice de temperatura-umidade e velocidade do ar (ITUV) (equação 1) são utilizados para determinar o $\Delta \mathrm{t}_{90}$ (aumento de temperatura corporal aos 90 minutos de exposição térmica) que, neste trabalho, foi representado por ATC. Empregando-se a equação proposta por Tao \& Xin (2003), o ATC pode ser calculado em função do ITUV, conforme a equação

$$
\text { ATC }=0,39 \cdot \text { ITUV }-12,22\left(R^{2}=0,847\right)
$$

Para análise do ambiente térmico, foi utilizado o intervalo de ATC, proposto por Tao \& Xin (2003) para classificação do estado de conforto dos frangos de corte, obtido com base em resultados experimentais (Quadro 4). Entretanto, a terminologia de classificação foi adaptada aos padrões brasileiros, de acordo com o proposto por Moraes e Oliveira (2007). Enquanto Tao \& Xin (2003) classificaram os intervalos de desconforto em normal, alerta, perigo e emergência, neste trabalho, as temperaturas do ar entre 15 e $26^{\circ} \mathrm{C}$ são consideradas como de conforto térmico para frangos de corte com idades superiores a 21 dias (CURTIS, 1983; ESMAY \& DIXON, 1986; FABRÍCIO, 1994; MEDEIROS, 2001); assim, valores superiores a $26^{\circ} \mathrm{C}$ caracterizam desconforto térmico. Oliveira et al. (2006) observaram que os melhores resultados de ganho de peso e dos pesos absolutos de carcaça, peito e coxa foram obtidos, quando as aves foram criadas em ambientes com temperaturas do ar entre $24^{\circ} \mathrm{C}$ e $26,3^{\circ} \mathrm{C}$.

Os valores de ATC medidos experimentalmente por Tao \& Xin (2003) foram obtidos pela diferença da temperatura corporal no final de cada teste, após a ave ter sido submetida a um ambiente de estresse agudo, e a temperatura corporal em condições termoneutras $\left(21 \pm 1,1^{\circ} \mathrm{C}\right.$ de $t_{b s}$ e $40 \pm$ $5 \%$ de UR). A temperatura corporal foi medida por meio de um sistema de telemetria, em que um sensor na forma de pílula era ingerido pelo frango de corte.

\section{RESULTADOS E DISCUSSÃO}

O Quadro 5 mostra os valores do ATC (ME), medidos experimentalmente, valores do ATC calculados por meio da equação proposta por Tao \& Xin (2003) (EQ) e os valores simulados por meio do sistema fuzzy (SF) proposto.

Os valores da ATC, estimados pelo sistema fuzzy, apresentaram menores valores absolutos de desvios, em relação aos valores da ATC calculados pela equação proposta por Tao \& Xin (2003), quando comparados àqueles obtidos experimentalmente, sendo que os desvios foram de $0,1^{\circ} \mathrm{C}$ e $0,3^{\circ} \mathrm{C}$, respectivamente.

Este menor valor de desvio absoluto, obtido por meio do sistema de inferência fuzzy, permitiu que a classificação do estado de estresse fosse mais realística, o que pode ser verificado no número de erros de classificação, obtidos pelo sistema fuzzy e pela equação proposta por Tao \& Xin (2003), que foram de 2 e 6, respectivamente. Estes erros de classificação são realçados, por meio das células preenchidas com fundo cinza no Quadro 5. No caso do sistema fuzzy, os únicos erros de classificação ocorreram para a combinação de $\mathrm{T}=35^{\circ} \mathrm{C}$, UR $=60 \%$ e V $=1,2$ $\mathrm{m} \mathrm{s}^{-1}$ e ainda, para $\mathrm{T}=41^{\circ} \mathrm{C}$, UR $=43 \%$ e V $=$ $1,2 \mathrm{~m} \mathrm{~s}^{-1}$. Enquanto o sistema fuzzy classificou o estado de estresse do animal como sendo severo desconforto, os dados experimentais indicaram uma situação de extremo perigo de vida, embora o desvio absoluto do ATC para esta condição ambiental ter sido de apenas 0,1 - $\mathrm{C}$, sendo que os resultados simulados pelo sistema fuzzy foram, estatisticamente, iguais aos medidos ( $P>0,05$, teste $t)$. 
Quadro 5. Comparação dos valores da variação da temperatura corporal de frangos de corte (ATC) e classificação do ambiente térmico obtida por meio de medições (ME), equação empírica (EQ) e sistema fuzzy (SF)

\begin{tabular}{|c|c|c|c|c|c|c|c|c|c|c|}
\hline \multicolumn{3}{|c|}{ Ambiente térmico } & \multicolumn{5}{|c|}{ ATC $\left({ }^{\circ} \mathrm{C}\right)$} & \multicolumn{3}{|c|}{ Classificação } \\
\hline $\begin{array}{c}t_{\mathrm{bs}} \\
\left({ }^{\circ} \mathrm{C}\right)\end{array}$ & $\begin{array}{l}\text { UR } \\
(\%)\end{array}$ & $\begin{array}{c}\mathrm{V} \\
\left(\mathrm{m} \cdot \mathrm{s}^{-1}\right)\end{array}$ & $\mathrm{ME}^{1}$ & $\mathrm{EQ}^{2}$ & $S F^{3}$ & Desvio $^{12}$ & Desvio $^{13}$ & $\mathrm{ME}^{1}$ & $\mathrm{EQ}^{2}$ & $\mathrm{SF}^{3}$ \\
\hline \multirow{6}{*}{35} & \multirow{3}{*}{40} & 0,2 & 1,5 & 2,1 & 1,8 & 0,4 & 0,2 & MD & MD & MD \\
\hline & & 0,7 & 1,0 & 1,1 & 0,6 & 0,0 & 0,3 & LD & MD & LD \\
\hline & & 1,2 & 0,5 & 0,6 & 0,5 & 0,1 & 0,0 & LD & LD & LD \\
\hline & \multirow{3}{*}{60} & 0,2 & 2,3 & 2,3 & 1,8 & 0,0 & 0,4 & $M D$ & $\mathrm{MD}$ & MD \\
\hline & & 0,7 & 1,2 & 1,3 & 1,1 & 0,1 & 0,1 & MD & MD & MD \\
\hline & & 1,2 & 1,0 & 0,9 & 1,1 & 0,1 & 0,1 & LD & LD & MD \\
\hline \multirow{6}{*}{38} & \multirow{3}{*}{34} & 0,2 & 2,3 & 3,2 & 2,2 & 0,6 & 0,1 & MD & SD & MD \\
\hline & & 0,7 & 2,0 & 2,1 & 1,8 & 0,1 & 0,1 & MD & MD & MD \\
\hline & & 1,2 & 1,5 & 1,7 & 1,8 & 0,1 & 0,2 & MD & MD & MD \\
\hline & \multirow{3}{*}{51} & 0,2 & 3,0 & 3,5 & 2,9 & 0,3 & 0,1 & SD & SD & SD \\
\hline & & 0,7 & 2,9 & 2,4 & 2,7 & 0,4 & 0,1 & SD & MD & SD \\
\hline & & 1,2 & 1,7 & 1,9 & 1,8 & 0,2 & 0,1 & MD & $M D$ & MD \\
\hline \multirow{7}{*}{41} & \multirow{3}{*}{29} & 0,2 & 3,9 & 4,3 & 3,6 & 0,3 & 0,2 & SD & EP & SD \\
\hline & & 0,7 & 3,7 & 3,2 & 3,6 & 0,4 & 0,1 & $S D$ & $S D$ & SD \\
\hline & & 1,2 & 3,4 & 2,7 & 3,6 & 0,5 & 0,1 & SD & SD & SD \\
\hline & \multirow{4}{*}{43} & 0,2 & 4,3 & 4,6 & 4,1 & 0,2 & 0,1 & EP & EP & EP \\
\hline & & 0,7 & 4,1 & 3,4 & 4,1 & 0,5 & 0,0 & EP & SD & EP \\
\hline & & 1,2 & 4,1 & 2,9 & 4,0 & 0,8 & 0,1 & EP & SD & SD \\
\hline & & & & & Média & 0,3 & 0,1 & & & \\
\hline
\end{tabular}

No caso específico das classificações feitas pela equação proposta por Tao \& Xin (2003), renomeadas de acordo com Moraes e Oliveira (2007), as classificações errôneas ficaram distribuídas ao longo das condições ambientais testadas.

Visando ilustrar uma possível aplicação do sistema fuzzy desenvolvido, foram simuladas condições ambientais entre 12 e 17 horas, a fim de classificar as condições de estresse sofrido por frangos de corte, submetidos a um dia com ocorrência de onda de calor severa (Figura 5). Os resultados da simulação mostram que, entre 12:00 e 12:13 horas e entre 16:42 e 17:00 horas, a condição de estresse das aves é considerada como leve desconforto, de
12:13 a 13:12 horas e de 15:22 a 16:42 horas é considerada como moderado desconforto e no intervalo de 15:22 a 16:42 horas assume-se um estado de severo desconforto. Considerando que a condição com moderado desconforto implicaria na tomada de alguma ação, o sistema fuzzy poderia ser usado para acionar um estágio mais avançado do sistema de ventilação e resfriamento evaporativo, sendo que, para o estado de perigo, algum sistema de resfriamento alternativo, como a aspersão de água diretamente sobre as aves, por exemplo, poderia ser acionado com o propósito de evitar maiores perdas ao plantel, conforme pesquisas desenvolvidas por Chepete \& Xin (2000), Ikeguchi \& Xin (2001) e Yanagi Junior et al. (2002). 


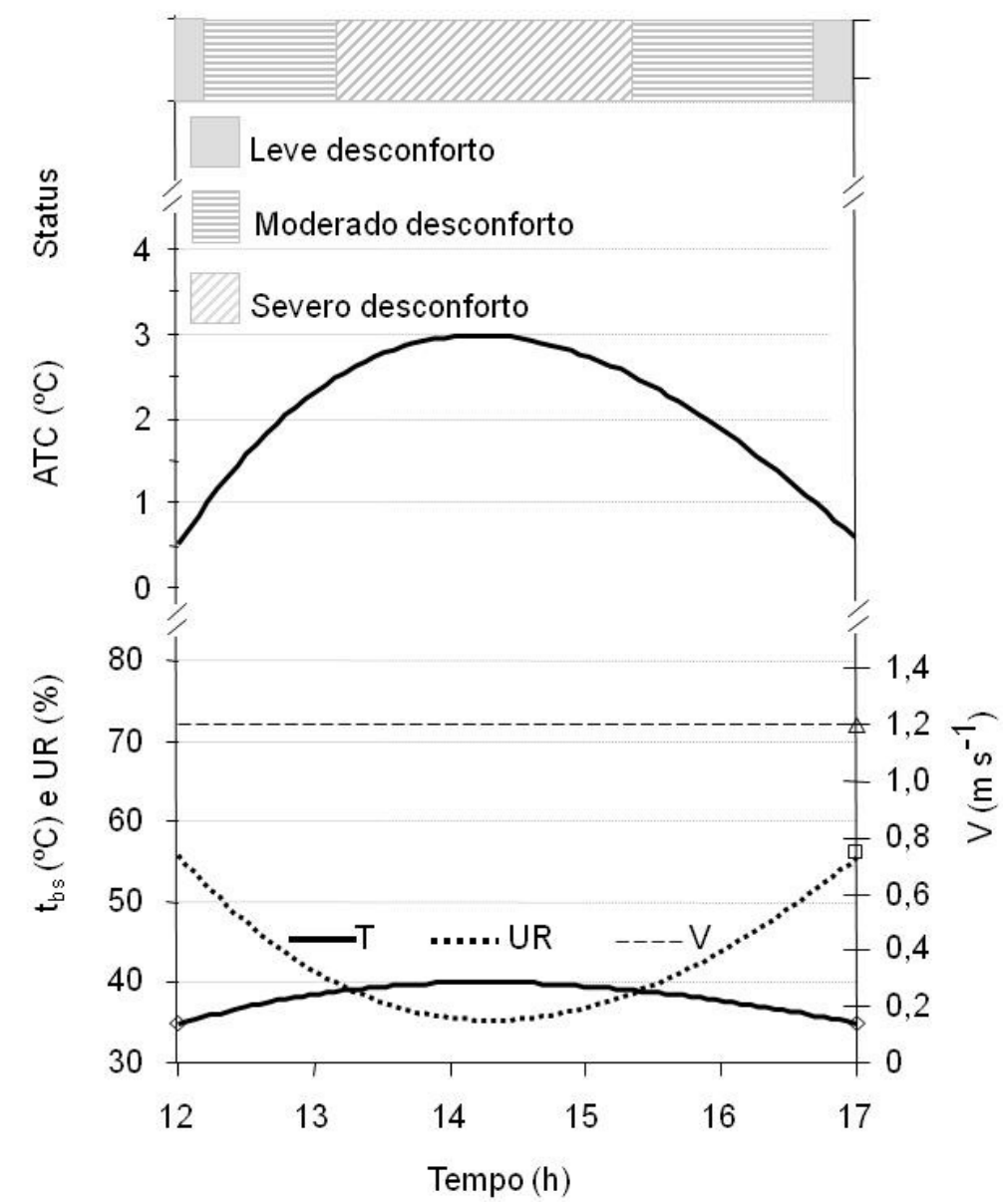

Figura 5. Simulação do estado de desconforto térmico de frangos de corte, submetidos a um dia caracterizado pela presença de uma onda de calor

\section{CONCLUSÕES}

Com base nos resultados, conclui-se que:

- O sistema fuzzy desenvolvido para a predição do aumento da temperatura corporal aos 90 min de exposição térmica (ATC), com base na temperatura $\left(\mathrm{t}_{\mathrm{bs}}\right)$, umidade relativa (UR) e velocidade do ar (V), proporcionou baixos valores de desvio absoluto, em relação àqueles obtidos experimentalmente $(0,1 \stackrel{\circ}{\circ})$, possibilitando uma classificação mais realística do estado de desconforto dos frangos de corte.

- A lógica fuzzy é uma tecnologia promissora na predição do ATC em frangos de corte, submetidos a estresse térmico agudo, podendo ser usada como suporte à tomada de decisão sobre o controle de sistemas de acondicionamento térmico de galpões avícolas.

\section{AGRADECIMENTOS}

Os autores expressam seus agradecimentos à FAPEMIG, à CAPES e ao $\mathrm{CNPq}$, pelo financiamento do projeto e pela concessão de bolsas de estudos aos estudantes.

\section{REFERÊNCIAS BIBLIOGRÁFICAS}

AMENDOLA, M.; CASTANHO, M.J.; NÄÄS, I.A. Análise matemática de conforto térmico para avicultura usando a teoria dos conjuntos fuzzy. Biomatemática, n.14, p. 87-92, 2004.

AMENDOLA, M.; NETO, M.M.; CRUZ, V.F. Using fuzzy sets to analyze environmental condition in order to improve animal productivity. Biomatemática, n.15, p. 29-40, 2005. 
AMENDOLA, M.; SOUZA, A.L. Manual do uso da teoria dos conjuntos fuzzy no MATLAB 6.1. Campinas: FEAGRI/UNICAMP, 2004. 30p.

CHAO, K.; GATES, R.S.; SIGRIMIS, N. Fuzzy logic controller design for staged heating and ventilating systems. Transactions of the ASAE, v.43, n.6, p. 1885-1894, 2000.

CHEPETE, H.J.; XIN, H. Cooling laying hens by intermittent partial surface sprinkling. Transactionsof the ASAE, St. Joseph, v.43, n.4, p.965-71, 2000.

CURTIS, S.E. Environmental management in animal agriculture. The lowa State University: Ames, 1983. 410 p.

CURTIS, S.E. Environmental management in animal agriculture. Ames: The lowa State University, 1983. 410 p.

DONALD, J. Environmental Control options under different climate conditions. World Poultry Elsevier, v. 14, n. 11, p. 22-27, 1998.

ESMAY, M.L.; DIXON, J.E. Environmental control for agricultural buildings. West port: AVI, 1986. 287 p.

FABRÍCIO, J.R. Influência do estresse calórico no rendimento da criação de frango de corte. In: CONFERÊNCIA APINCO DE CIÊNCIA E TECNOLOGIA AVÍCOLA, 1994, São Paulo. Anais... São Paulo: Apinco, 1994, p. 129-133.

FERREIRA, L.; YANAGI JUNIOR, T.; NÄÄS, A.I.; LOPES, M.S. Predição de cio em vacas leiteiras utilizando lógica fuzzy. In: CONGRESSO BRASILEIRO DE ENGENHARIA AGRíCOLA, 35, 2006, João Pessoa. Anais... João Pessoa: SBEA, 2006.

FIRK, R.; STAMER ,E.; JUNGE, W.; KRIETER, J. Improving oestrus detection by combination of activity measurements with information about previous oestrus cases. Livestock Production Science, n. 82, p. 97-103, 2003.

GATES, R.S.; CHAO, K.; SIGRIMIS, N. Identifying design parameters for fuzzy control of staged ventilation control systems. Computers and Eletronics in Agriculture, v.31, p.61-74, 2001.

IKEGUCHI, A.; XIN, H. Field evaluati on of a sprinkling system for cooling commercial laying hens in lowa. Applied Engineering in Agriculture, St. Joseph, v. 17, n.2, p.217221, 2001.

KLIR, G.J.; YUAN, B. Fuzzy sets and fuzzy logic. New Jersey: Prentice Hall PTR, 1995. $574 p$.

LOPES, G.T. Proposta de um controlador ótimo de altura da plataforma de corte de colhedoras. Campinas: UNICAMP, 1999. 155p. (Tese de doutorado)

MENDEL, J.M. Fuzzy logic systems for engineering: A tutorial. IEEE, v. 83, n. 03, p. 345-377, 1995.

MEDEIROS, C.M. Ajuste de modelos e determinação de índice térmico ambiental de produtividade para frangos de corte. 2001. 115 p. Tese (Doutorado em Engenharia Agrícola) - Universidade Federal de Viçosa, Viçosa, MG.

MORAES, S.R.P. ; OLIVEIRA, A.L.R. Classificação das faixas do índice de temperatura e umidade (ITU), aptidão da região e condições de conforto para frangos de corte e poedeiras, no Brasil. In: XV Congresso Brasileiro de Agrometeorologia, 2007, Aracaju - SE. Anais... XV Congresso Brasileiro de Agrometeorologia. Aracaju SE: CBAGRO, 2007. v.I. (CD-ROM).

NATIONAL WEATHER SERVICE CENTRAL REGION. Livestock hot weather stress. Regional Operations Manual Letter, C-3176. 1976. 
OLIVEIRA, H. L.; AMENDOLA, M.; NÄÄS I. A. Estimativa das condições de conforto térmico para avicultura de postura usando a teoria dos conjuntos fuzzy. Engenharia Agrícola, v. 25, n.2, p.300-307, 2005.

OLIVEIRA, G.A.; OLIVEIRA, R.F.M.; DONZELE, J.L.; CECON, P.R.; VAZ, R.G.M.V.; ORLANDO, U.A.D. Efeito da temperatura ambiente sobre o desempenho e as características de carcaça de frangos de corte dos 22 aos 42 dias. R. Bras. Zootec., v.35, n.4, p.1398-1405, 2006.

ORTEGA, N.R.S. Aplicação da teoria de conjuntos fuzzy a problemas da biomedicina. São Paulo: USP, 2001. 152p. (Tese de doutorado)

PEDRYCZ, W.; GOMIDE, F. An introduction to fuzzy sets: analysis and design. Cambridge: MIT Press, 1998. 461 p.

QUEIROZ, M.; NÄÄS, I.A.; SAMPAIO, C. Estimating thermal comfort of piglets considering ammonia concentration. Agricultural Engeneering International: the CIGR Ejournal, Manuscript IT 05 004/BC 05 005, v. 03, 2005.

RIBACIONKA, F. Sistemas computacionais baseados em lógica fuzzy. São Paulo: Universidade Mackenzie, 1999. 115p. (Tese de mestrado)
TANAKA, K. An introduction to fuzzy logic for practical applications. Tokyo: Springer, 1997. 138p.

TAO, X.; XIN, H. Acute synergistic effects of air temperature, humidity, and velocity on homeostasis of market-size broilers. Transactions of the ASAE, v. 46, n. 2, p. 491-497, 2003.

THOM, E.C. The discomfort index. Weatherwise, v. 12, n. 1, p. 57-60, 1959.

WEBER, L.; KLEIN, P.A.T. Aplicações de lógica fuzzy em software e hardware. Canoas: Editora ULBRA, 2003. 112p.

YANAGI JUNIOR, T.; XIN, H.; GATES, R.S. . Optimization of partial surface wetting to cool caged laying hens. Transactions Of The ASAE, St. Joseph, v. 45, n. 5, 2002.

YANAGI JUNIOR, T.; XIN, H.; GATES, R.S.; FERREIRA, L. Fuzzy logic model to predict laying hen body temperature rise during acute heat stress. In: CONGRESSO BRASILEIRO DE ENGENHARIA AGRíCOLA, 35, 2006, João Pessoa. Anais... João Pessoa: SBEA, 2006.

ZADEH, L.A. Fuzzy Sets. Journal Information and Control, n. 8, p. 338-353, 1965. 